\title{
Morbus Adamantiades-Behçet: Epidemiologie, pathogenetische Konzepte und therapeutische Möglichkeiten
}

\author{
A. Altenburg ${ }^{1,4}$ \\ N. Papoutsis ${ }^{2}$ \\ H. Orawa ${ }^{3}$ \\ P. Martus ${ }^{3}$ \\ C. C. Zouboulis ${ }^{1,2,4}$
}

\author{
Adamantiades-Behçet's Disease: Epidemiology, Pathogenetic Concepts and \\ Therapeutic Possibilities
}

\section{Zusammenfassung}

Morbus Adamantiades-Behçet (MAB) ist eine seltene polysymptomatische Erkrankung, die durch periodische Exazerbationen oraler Aphthen, Genitalulzera, unterschiedliche kutane Läsionen, Uveitis und eine Vielzahl anderer Organmanifestationen charakterisiert ist. Die Diagnosestellung erfolgt anhand nicht einheitlicher Kriterien und erfordert multidisziplinäres Fachwissen. Obwohl eine frühe Therapie oft von entscheidender prognostischer Bedeutung ist, erfolgt die Diagnosestellung oft mit einer Verzögerung von mehreren Jahren. Bleibender Visusverlust, Meningoenzephalitis, Thrombosen, pulmonale Beteiligung und Darmperforation gehören zu den möglichen schweren Komplikationen. Die genaue Ätiologie der Erkrankung, deren Manifestation von genetischer Prädisposition und Umwelteinflüssen abzuhängen scheint, ist unbekannt. Eine symptom- und patientengerechte Therapie kann den Verlauf jedoch günstig beeinflussen oder zur Vollremission führen. In Deutschland widmet sich das „Deutsche Register Morbus Adamantiades-Behçet e.V.“ der Erforschung der Epidemiologie, des klinischen Verlaufs der Erkrankung, derTherapie und der Förderung der Wissenschaft auf dem Gebiet des MAB.
Abstract

Adamantiades-Behçet's disease (ABD) is a rare polysymptomatic illness, which is characterized by periodical recurrence of oral aphthous ulcers, genital ulcers, different skin lesions, uveitis and a variety of other organ manifestations. Diagnosis is set through application of non-uniform criteria and requires multidisciplinary specialized knowledge. Although an early treatment is often of decisive prognostic importance, diagnosis is often carried out with a delay of several years. Lasting visual loss, meningoencephalitis, thromboses and pulmonary and intestinal perforation count to the severe complications. The exact etiology of the disease, whose manifestation seems to be depended on a genetic predisposition and environmental influence, is unknown. Symptomatic and patient-tailored therapy can influence favourably the course of the disease and even lead to the full remission. The „German Register Morbus Adamantiades-Behçet e.V.“ devotes itself to the study of epidemiology, clinical course and therapy of the illness in Germany and the promotion of the science in the field of ABD.

\section{Epidemiologie}

Das „Deutsche Register Morbus Adamantiades-Behçet e.V.“ wurde 1990 gegründet und 2001 als gemeinnütziger eingetragener Verein anerkannt. Es hat seinen Sitz am Städtischen Klinikum
Dessau. Bis zum Juni 2006 waren 640 Patienten mit festem Wohnsitz in Deutschland aus 15 medizinischen Zentren sowie aus zahlreichen Praxen dem Register gemeldet worden. Das Städtische Klinikum Dessau ist auch Sitz einer Sondersprechstunde für Patienten mit MAB.

Institutsangaben

${ }^{1}$ Hautklinik und Immunologisches Zentrum, Städtisches Klinikum Dessau, Dessau

${ }^{2}$ Forschergruppe für Biogerontologie, Dermato-Pharmakologie \& Dermato-Endokrinologie, Institut für Klinische Pharmakologie \& Toxikologie, Charité Universitätsmedizin Berlin, Campus Benjamin Franklin, Berlin

${ }^{3}$ Institut für Medizinische Informatik, Biometrie und Epidemiologie, Charité Universitätsmedizin Berlin, Campus Benjamin Franklin, Berlin

${ }^{4}$ Deutsches Register Morbus Adamantiades-Behçet e.V., Dessau

Korrespondenzadresse

Prof. Dr. med. Christos C. Zouboulis · Hautklinik und Immunologisches Zentrum • Städtisches Klinikum Dessau · Auenweg 38 · 06847 Dessau · E-mail: christos.zouboulis@klinikum-dessau.de

Bibliografie

Akt Dermatol 2006; 32: 360-364 @ Georg Thieme Verlag KG Stuttgart · New York

DOI $10.1055 / \mathrm{s}-2006-944658 \cdot$ ISSN 0340-2541 
Von den zwischen 1961 und 2005 diagnostizierten Patienten waren 227 (38,5\%) deutscher und 267 (45,3\%) türkischer Abstammung, jeweils 10 (1,7\%) kamen aus Griechenland, Italien und dem Libanon und weitere 66 aus 27 anderen Ländern. Auf der Grundlage des Registers kann eine Krankheitshäufigkeit von zumindest 1,0 auf 100000 Einwohner in Deutschland angenommen werden. Der Morbus Adamantiades-Behçet tritt vermehrt im östlichen Mittelmeerraum, im Mittleren und Fernen Osten auf. Endemieländer mit der häufigsten Prävalenz sind die Türkei, Saudi-Arabien, der Iran, China und Nord-Japan. In nord- und westeuropäischen Ländern ist die Erkrankung 10- bis über 100-mal seltener. Die weltweit höchste Prävalenz besteht in Anatolien (nordöstliche Türkei) mit 370 Patienten pro 100000 Einwohner [1].

Die Erkrankung manifestierte sich in unserem Kollektiv überwiegend im 3. Lebensjahrzehnt (Medianalter 26 Jahre) mit einer großen Spannbreite. Der volle Symptomenkomplex entwickelte sich nach 3 Monaten (Medianwert) mit großer Spannbreite von bis zu 38 Jahren. Die Diagnose wurde erst mit einer durchschnittlichen Verzögerung von mehr als 2 Jahren nach der Entwicklung eines vollen klinischen Bildes gestellt. Bei 13\% der Patienten kam es zur Erstmanifestation in der Kindheit, meistens mit oralen Aphthen, und bei $4 \%$ zur Ausprägung des vollen Symptomenkomplexes vor dem 16. Geburtstag. Im Gesamtkollektiv ließ sich keine Bevorzugung eines Geschlechts beobachten, jedoch lag bei Patienten türkischer Herkunft eine Androtropie vor (Männer : Frauen türkischer Herkunft: 1,9:1; $<$ <,001).

\section{Klinische Symptomatik}

\section{Hauptsymptome}

Orale Aphthen traten bei 98,5\% der Patienten in Deutschland und als häufigstes Initialsymptom bei 83,8\% auf. Orale Aphthen werden als mit Fibrin belegte, scharf begrenzte schmerzhafte Ulzerationen mit umgebendem Erythem charakterisiert. Die klinischen Stadien der Aphthose sind in Tab. 1 dargestellt [2]. Kleine Ulzera heilen meist ohne Narbenbildung (8\%), lediglich große Ulzera heilen in bis zu 64\% mit Narbenbildung ab (Abb.1 u.2). In schweren Fällen können Probleme wie Dysphagie und Dyspnoe, auch Fisteln an Pharynx oder Larynx beobachtet werden.

Genitalulzera wurden bei 63,7\% der Patienten gesehen und sind das häufigste zweite Symptom. Die oft wie ausgestanzt erscheinenden, am Grund mit fibrinösem Exsudat oder einer Kruste belegten, schmerzhaften Ulzera rezidivieren in der Regel weniger häufig als die oralen Aphthen, sind von längerer Persistenz und besitzen häufiger die Tendenz zur Narbenbildung. Sie können neben der Genitalregion im Bereich des Anus, des Perineums und der Inguinalregion auftreten (Abb. 3).

Hautmanifestationen treten bei 81,3\% der Patienten auf. Papulopustulöse Veränderungen lassen sich bei 62,5\% (Abb. 4), Pyodermie bei $14,9 \%$, Hautulzera bei $12,8 \%$ und superfizielle Thrombophlebitis bei 13,0\% der Patienten finden. Seltener können nekrotisierende Vaskulititiden, Erythema-exsudativum-multiforme- und Sweet-artige Läsionen gesehen werden.
Tab. 1 Phasen der oralen Aphthose

\begin{tabular}{|c|c|}
\hline Phasen der oralen Aphthose & Dauer \\
\hline $\begin{array}{l}\text { Prodromalstadium: } \\
\text { Kribbeln, Spannungsgefühl, Brennen, Rauigkeit }\end{array}$ & 24 Stunden \\
\hline $\begin{array}{l}\text { Präulzeröse Phase: } \\
\text { inflammatorisches Erythem oder indurierte Papel }\end{array}$ & 1-3 Tage \\
\hline $\begin{array}{l}\text { Ulzeratives Stadium: } \\
\text { fibrinbelegte Ulzeration mit aufgeworfenem } \\
\text { erythematösem Rand }\end{array}$ & 1-16 Tage \\
\hline Abheilungsphase & 4 Tage - 1 Monat \\
\hline
\end{tabular}

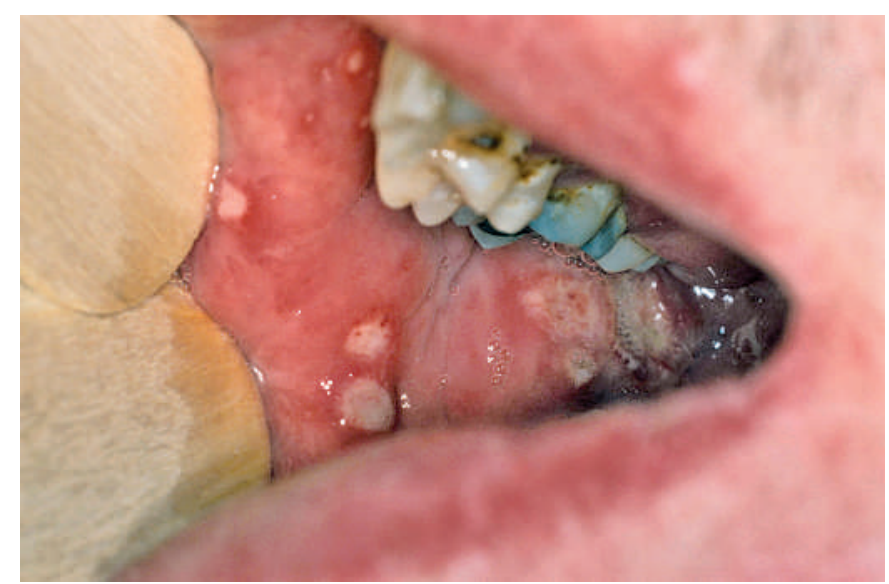

Abb. 1 Orale Aphthen.

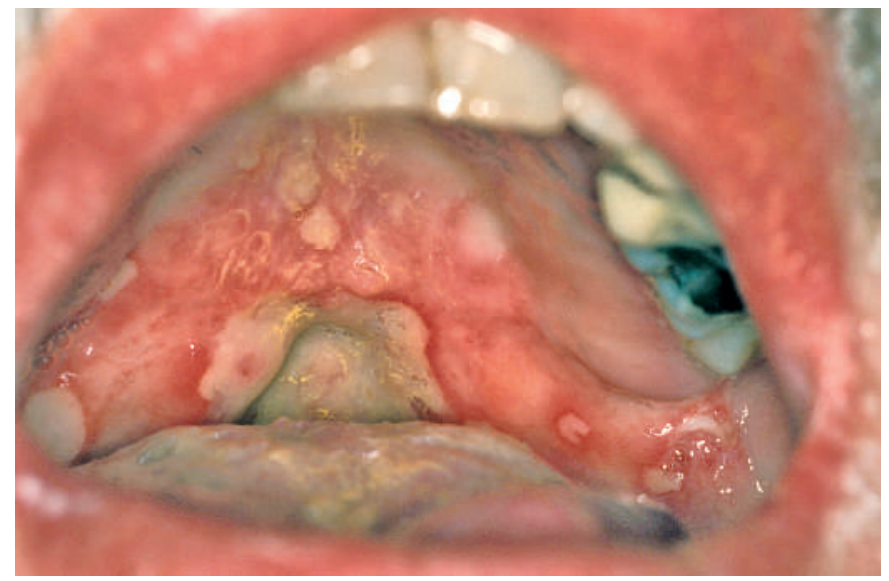

Abb. 2 Große orale Aphthen am tieferen Mundabschnitt.

Die Augenmanifestation gehört zu den vier häufigsten klinischen Manifestationen (58,1\%). Die Hypopyon-Iritis galt früher als Leitsymptom der Krankheit. Bei den meisten Patienten zeigt sich jedoch eine Panuveitis (Abb.5) sowie eine retinale Vaskulitis und Chorioiditis, wobei der Vorderkammerreiz fehlen kann [3].

Eine objektivierbare Arthritis lässt sich bei 53\% der Patienten in Deutschland konstatieren. Gelenkmanifestationen treten häufig zuerst an einem Knie bzw. Sprunggelenk, dann am anderen als Monoarthritis saltans, an beiden Gelenken gleichzeitig und anschließend an fast allen Gelenken auf. 


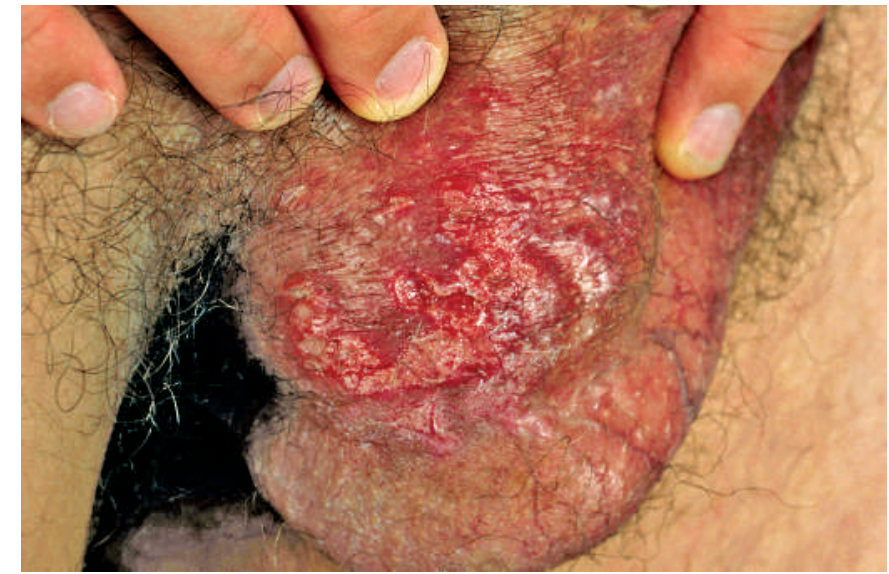

Abb. 3 Skrotalulzera.



Abb. 5 Uveitis.

Bei der Beteiligung größerer Gefäße, die in 22,7\% unserer Patienten auftrat, handelte es sich oft um eine rezidivierende Thrombose der superfiziellen oder tiefen Venen und nur selten um einen arteriellen Befall in Form von Thrombosen oder Aneurysmen.

\section{Andere Manifestationen}

Aseptische Meningitis, z. T. mit Enzephalitis, eine vaskuläre zerebrale Beteiligung oder Pyramidenbahnstörung ließen sich bei 10,9\% der Patienten finden. Eine neurologische Manifestation kündigt sich meist mit schweren Kopfschmerzen an. Weitere Symptome wie Gangunsicherheit, Dysarthrie, Diplopie, epileptische Anfälle, positiver Babinski-Reflex und auch psychiatrische Symptome, wie Depression oder Gedächtnisschwierigkeiten, sind hinweisend. Mit der Magnetresonanztomographie kann eine ZNS-Beteiligung objektiviert werden.

Gastrointestinale Beschwerden, einhergehend mit Gastritis, Ösphagitis, Duodenitis oder Colitis sowie gastrointestinalen Ulzerationen, traten bei $11,6 \%$ unserer Patienten auf.

Eine sterile Prostatitis oder Epididymitis konnte bei 8,8\% der männlichen Patienten, eine Lungenmanifestation mit Husten

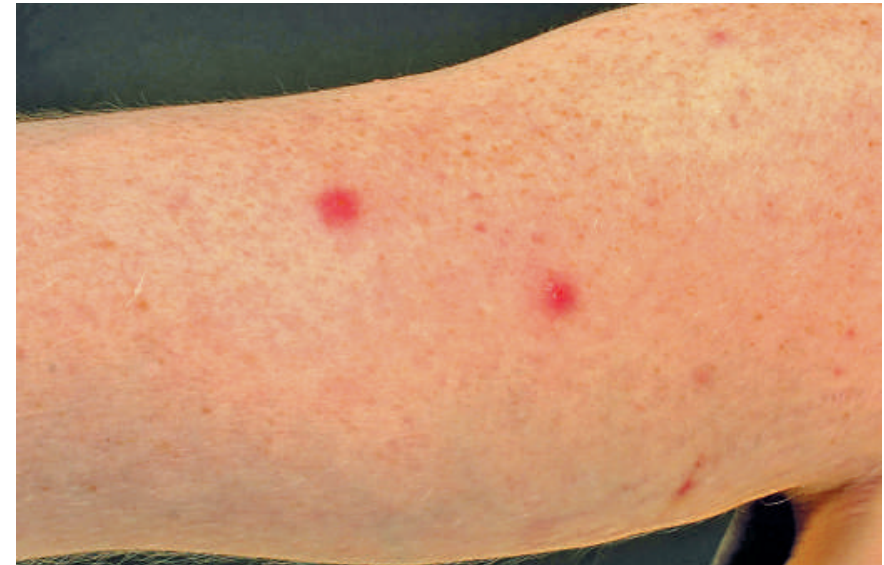

Abb. 4 Papulopusteln.

und Hämoptyse bei 3,6\% konnte etwa so häufig wie eine Herzmanifestation (3,2\%) gesehen werden. Eine Glomerulonephritis als Ausdruck einer Nierenvaskulitis war insgesamt selten (2,2\%).

Schwere Komplikationen ergaben sich bei $12 \%$ der Patienten (Erblindung 8,6\%, Meningoenzephalitis 4,7\%, schwere Arthritis $3,3 \%$, letaler Ausgang 1,4\% [ $n=6$ ], Hämoptyse 1,2\%, Darmperforation $0,5 \%[n=2])$.

Ein positives Pathergiephänomen manifestiert sich innerhalb 48 Stunden als erythematöse Papel $(>2 \mathrm{~mm}$ ) oder Pustel am Ort eines Nadelstiches, der 3-5 mm intrakutan in einem Winkel von $45^{\circ}$ am volaren Unterarm mit einer 20-Gauge-Kanüle ohne Hautdesinfektion gesetzt wird, oder einer intrakutanen Injektion von $0,1 \mathrm{ml}$ isotoner Kochsalzlösung. Der Pathergietest fiel bei 33,7\% der Patienten positiv aus [4].

\section{Histopathologie}

Primär handelt es sich um eine Vaskulitis, wobei die kleinen und sehr kleinen Gefäße befallen werden. Die Aneurysmabildung in den großen Arterien wird auch durch eine Vaskulitis der Vasa vasorum und damit einen Durchbruch der Lamina elastica verursacht. Eine leukozytoklastische Vaskulitis wurde bei allen mukokutanen Läsionen beschrieben. Im ulzerösen Stadium ist das Ulkus mit fibrinösem Belag und Granulozytenrasen belegt. Weiterhin kommt es zur fibrinoiden Nekrose des Endothels und ggf. zu tiefreichenden Gewebsnekrosen.

\section{Diagnosekriterien}

Im Jahre 1953 legten Adamantiades und 1969 Mason und Barnes die ersten Diagnosekriterien fest. Nach den letzteren werden 3 Hauptkriterien oder 2 Haupt- und 2 Nebenkriterien benötigt. Die Klassifikationskriterien der „International Study Group for Behçet's Disease“" wurden seit 1990 häufig als Diagnosekriterien herangezogen [5]. Ein obligates Symptom, die orale Aphthose, und mindestens zwei weitere Symptome sind hiernach nötig (Tab. 2). Davatchi et al. fertigten 1993 das System des „Classification and Regression Tree“ (CART-Methode) an (Abb. 6) [6]. Orale Aphthen sind danach nicht obligat und mindestens zwei Symp- 


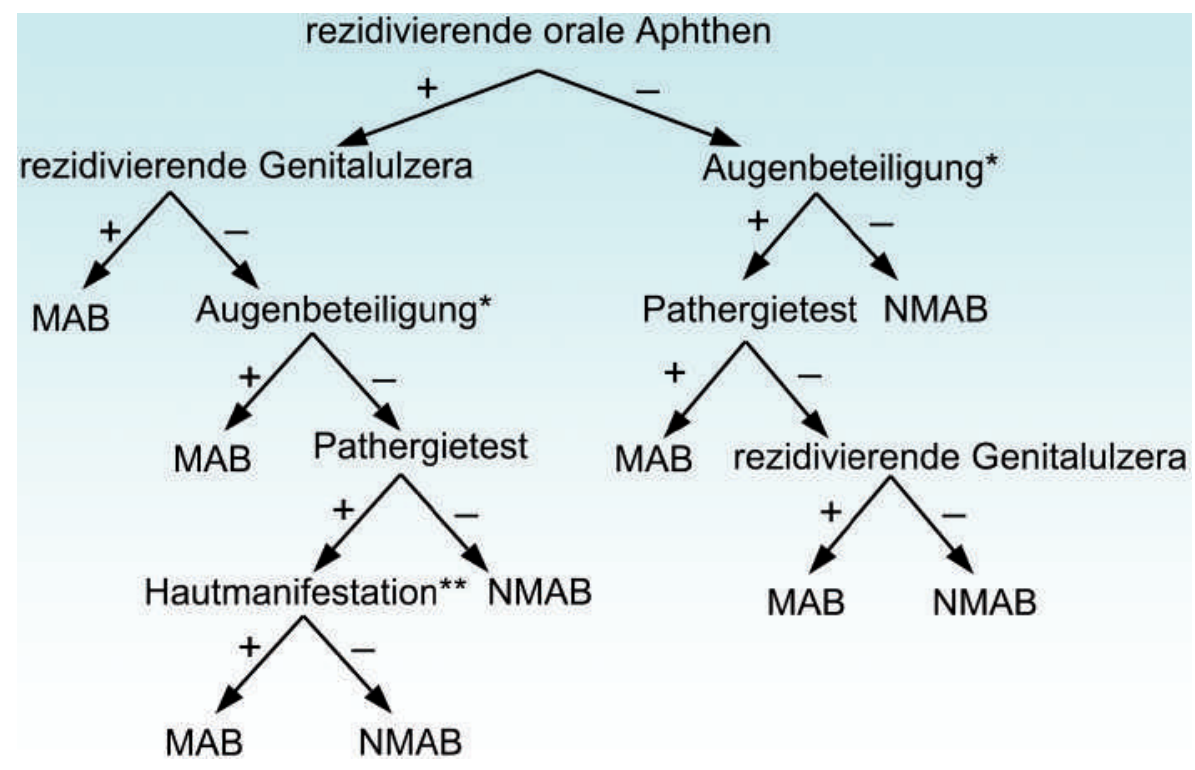

Abb. 6 Anwendung der CART-Methode zur Diagnose des Morbus AdamantiadesBehçet.

MAB: Morbus Adamantiades-Behçet

* Augenbeteiligung: Hypopyon-Iritis, Uveitis

** Hautmanisfestation: Papulopusteln, Erythema nodosum, superfizielle Thrombophlebitis.

Tab. 2 Klassifikationskriterien der „International Study Group for
Behçet’s Disease“

Kriterien der „International Study Group for Behçet's Disease“

obligates Symptom: rezidivierende orale Aphthen (mindestens 3-mal jährlich)

plus mindestens zwei der folgenden Kriterien:

- Genitalulzera

- Augenbeteiligung

- Hautveränderungen (Erythema nodosum, Follikulitis, sterile Pusteln, aphthöse Ulzerationen)

- positiver Pathergie-Test

tome werden für die Diagnose gefordert. In einer Studie unserer Klinik mit 70 Patienten mit MAB und 30 Kontrollpatienten, die an erosiven Schleimhautveränderungen oder entzündlichen Augenerkrankungen, dabei aber nicht an einem MAB erkrankt waren, konnten wir den „Cut-off-Point“ für die Diagnosestellung mit der höchsten Sensitivität und Spezifität bei einer 3-Symptomen-Konstellation (Sensitivität $=95 \%$, Spezifität $=90 \%$ ) ermitteln [7]. Eine Diagnosestellung anhand von nur zwei Symptomen hätte zu höheren falsch positiven (Spezifität $=70 \%$ ), mit wenigstens vier Symptomen zu mehr falsch negativen Diagnosen (Sensitivität $=61,4 \%$ ) geführt. Folgende Symptome hatten wir berücksichtigt: orale und genitale Aphthen-ähnliche Läsionen, Follikulitis, Erythema nodosum, Hautulzera, intraokuläre Entzündungen, Beteiligung des peripheren und zentralen Nervensystems, Thrombose, superfizielle Thrombophlebitis, Epididymitis, Pathergietest. Wir favorisieren also eine Konstellation von drei Symptomen, obwohl wir die Diagnose mit zwei Symptomen einschließen, wenn alle anderen infrage kommenden Erkrankungen in fraglichen Fällen - z. B. mittels Antikörpern gegen bullöse Dermatosen oder Herpes-PCR - ausgeschlossen werden können.

\section{Pathogenese}

\section{Genetische Prädisposition}

In Deutschland ließ sich eine positive Familienanamnese bei 8,9\% der Patienten ermitteln mit signifikantem Unterschied zwischen Patienten deutscher $(2,3 \%)$ und türkischer Herkunft (14,2\%; p <0,001). Das HLA-B5-Antigen besaßen 48,3\% der Patienten, unter den deutschen Patienten $36,6 \%$ und unter denen türkischer Herkunft 66,4\% ( $p<0,001)$. Unter der deutschen Gesamtbevölkerung beträgt die HLA-B5-Frequenz hingegen nur ca. $14 \%$ und in der Türkei je nach Region $23-33 \%$. Hinsichtlich einer signifikanten Assoziation mit einer Augenmanifestation stellt HLA-B5 einen Marker für eine ungünstigere Prognose dar [8]. Mehr als HLA-B51 selbst scheinen benachbarte Sequenzen auf Chromosom 6 in die Ätiologie der Erkrankung involviert zu sein, wie das MICA6-Allel oder die Tau-A Mikrosatelliten-Sequenz.

\section{Infektionen und immunologische Faktoren}

Die S.-Stämme sanguis und oralis, welche die Flora der oralen Schleimhaut der MAB-Patienten dominieren, scheinen relevante Bakterien zu sein, die die Initiierung der Erkrankung provozieren [9]. Mycoplasma fermentas wird mit rheumatoiden Erkrankungsbildern in Verbindung gebracht. Das M.-fermentas-Lipoprotein MALP-404 konnte in 32\% der Seren von MAB-Patienten, aber in keinem Fall der Kontrollpatienten detektiert werden [10]. MALP-404 enthält ein Peptidmotiv, welches auch von HLA-B51 präsentiert wird. Antigenepitope bakterieller Hitzeschockproteine (HSP) von S. sanguis und Mykobakterien besitzen Homologien mit humanem HSP und könnten im Sinne einer Kreuzreaktivität eine Rolle bei der autoimmunologischen Pathogenese spielen. Erhöhte Werte von anti-HSP-Antikörpern wurden beispielsweise im Liquor von Patienten mit ZNS-Beteiligung gefunden. Mehrfach wurde auch über erhöhte Konzentrationen proinflammatorischer Th1-Zytokine wie TNF- $\alpha$, Interferon- $\gamma$, IL-1, IL-12 und IL-6 im Serum oder im Liquor berichtet. Erhöhte Serumwerte von IL-18 bei MAB-Patienten in der akuten Krankheitsphase bekräftigten die Rolle einer Th1-dominierten Immunantwort [11]. 
Therapiemöglichkeiten

\section{Topische Behandlung}

Bei einzelnen Aphthen kommen lokale Anästhetika, z. B. Lidocain $2 \%$ in Gel oder als Spray, sowie antiseptische Mundspülungen, z. B. 0,1-0,2\%ige Chlorhexidin-Lösung, infrage. Die Kombination eines lokalen Anästhetikums tagsüber mit einer Triamcinolonhaftsalbe zur Nacht hat sich bewährt. Eine jeweils vor der Anwendung zubereitete Tetracyclin-Mundspülung (Tetracyclinhydrochlorid $250 \mathrm{mg}$ in $5 \mathrm{ml}$ Trinkwasser für 2 Minuten $2-4 \times /$ Tag) kann Dauer und Schmerzhaftigkeit der Aphthen verringern. Eine Sucralfatsuspension ( $4 \times 5 \mathrm{ml} /$ Tag) erwies sich ebenfalls als wirksam. Die Ätztherapie mit Silbernitratlösung (1-2\%) oder Wasserstoffperoxidlösung $(0,5 \%)$ ist eine altbewährte Methode. Insbesondere bei klarem anamnestischem Zusammenhang sollten folgende Nahrungsmittel und Hygienemittel gemieden werden: harte Speisen (z.B. Zwieback), Nüsse, saure oder gewürzte Speisen (Obstsäfte, Zitrusfrüchte, Pfeffer, Paprika), alkoholische oder $\mathrm{CO}_{2}$-haltige Getränke, natriumlaurysulphathaltige Produkte. Genitalulzera können mit einer Kombination fluorierter Kortikosteroide und Antiseptika in einer Cremegrundlage therapiert werden [12].

\section{Systemische Therapie}

Kortikosteroide: Prednisolon wird häufig bei akuter Exazerbation, einschließlich Uveitis und neurologischer Manifestation, eingesetzt, in der Regel in Kombination mit anderen Immunsuppressiva, Colchicin oder Diaminodiphenylsulfon (DADPS).

Colchicin und DADPS: Colchicin 1,0 bis 2,0 mg/Tag und DADPS $100-150 \mathrm{mg} /$ Tag (intermittierende Gabe von Ascorbinsäure ist während der Behandlung sinnvoll) können bei mukokutaner Symptomatik, insbesondere bei oraler und genitaler Aphthose, beim Erythema nodosum, oder bei Arthritis neben nicht-steroidalen Antiphlogistika eingesetzt werden.

Sulfasalazin: Sulfasalazin allein oder in Kombination mit Kortikosteroiden p.o. ist die Basistherapie bei Ulzerationen des Gastrointestinaltraktes.

Azathioprin: Azathioprin, allein oder kombiniert mit anderen Immunsuppressiva, kann die Inzidenz, Frequenz und Schwere der Augenmanifestation reduzieren. Günstige Effekte auf die orale und genitale Aphthose wurden beschrieben.

Calcineurininhibitoren (Cyclosporin-A und Tacrolimus): Cyclosporin-A ist zur Behandlung der MAB-assoziierten Uveitis in Deutschland zugelassen und kann die Schwere und Frequenz der Uveitis in Dosierungen von 3-6 mg/kg/Tag (kurzfristig bis $10 \mathrm{mg} / \mathrm{kg} / \mathrm{Tag}$ ), aber auch die mukokutanen Symptome, Thrombophlebitis, Hörsturz und weitere systemische Komplikationen günstig beeinflussen. Tacrolimus $0,05-0,2 \mathrm{mg} / \mathrm{kg} / \mathrm{Tag}$ p. o. wurde erfolgreich bei der refraktären Uveitis eingesetzt.

Alkylierende Substanzen (Cyclophosphamid und Chlorambucil): Cyclophosphamid ist in Form einer Pulstherapie mit $1000 \mathrm{mg} /$ Woche als i.v.-Bolusgabe neben hoch dosierten Kortikosteroiden ein wirksames Medikament bei neurologischen Verlaufsformen. Chlorambucil (6-8 mg/Tag p. o. und $2 \mathrm{mg} / \mathrm{kg}$ alle 1 bis 2 Tage als Erhaltungsdosis) ist ein Alternativpräparat bei neurologischer
Manifestation und auch gegen mukokutane und okuläre Symptomatik wirksam. Insbesondere wirksam hat sich Chrorambucil bei der Gefäßmanifestation der Erkrankung erwiesen.

Interferon-alpha: Interferon-alpha wurde bei der Behandlung mukokutaner Varianten der Erkrankung, Arthritis und vaskulärer Beteiligung erfolgreich eingesetzt. In hartnäckigen Fällen besserten sich die Symptome innerhalb von 1-4 Monaten. Insbesondere bei Augenbefall lässt sich eine langfristige Visusverbesserung beobachten. Wir empfehlen 6-9 Mio. I.E. $3 \times /$ Woche über 6 Monate und anschließend 3 Mio. I.E. $3 \times /$ Woche s. c. als Erhaltungstherapie.

Biologics: Infliximab wird in einer Dosierung von $5 \mathrm{mg} / \mathrm{kg}$ intravenös als akute Behandlung der Uveitis eingesetzt. Ein rasches Ansprechen von schwerer Uveitis, gastrointestinaler Manifestation, schweren mukokutanen Manifestationen und zerebraler Vaskulitis wurde teilweise schon nach der ersten Infusion auch bei Patienten gesehen, die gegenüber herkömmlichen Immunsuppressiva refraktär waren. Bei der Gabe von nachfolgenden Infusionen als Erhaltungstherapie wurde über rezidivfreie Intervalle von 2 bis 6 Monaten berichtet. Etanercept scheint sich günstig auf orale Aphthen, Erythema und Follikulitiden auszuwirken [4]. Aktuell wird lediglich Infliximab als Einzelinfusion bei akuter Uveitis zusätzlich zu der individuellen Therapie empfohlen.

\section{Literatur}

${ }^{1}$ Zouboulis CC. Epidemiology of Adamantiades-Behçet's Disease. In: Zierhut M, Ohno S. Immunology of Behçet's Disease. Lisse: Swets \& Zeitlinger Publishers, 2003: 1 - 16

2 Zouboulis CC. Morbus-Adamantiades-Behçet. Klinische und experimentelle Befunde von 53 Patienten aus dem Berliner Raum. Habilitationsschrift. Berlin: Freie Universität, 1994

${ }^{3}$ Krause L. Morbus Adamantiades-Behçet. Ophthalmologe 2005; 102 : $329-334$

${ }^{4}$ Altenburg A, Papoutsis N, Orawa H, Martus P, Krause L, Zouboulis CC. Epidemiologie und Klinik des Morbus Adamantiades-Behçet in Deutschland - Aktuelle pathogenetische Konzepte und therapeutische Möglichkeiten. J Dtsch Dermatol Ges 2006; 4: 49-64

${ }^{5}$ International Study Group for Behçet's Disease. Criteria for diagnosis of Behçet's disease. Lancet 1990; 335: 1078 - 1080

${ }^{6}$ Davatchi F, Shahram F, Akbarian M, Gharibdoost F, Nadji A, Chams C et al. Accuracy of existing diagnosis citeria for Behçet's disease. In: Wechsler B, Godeau P (eds). Behçet's disease. Amsterdam, London, New York, Tokyo: International Congress Series 1037, Excerpta Medica, 1993: $225-228$

${ }^{7}$ Altenburg A, Orawa H, Papoutsis N, Assaf C, Zouboulis CC. Evaluation of diagnostic criteria in Adamantiades-Behçet's in Germany. Exp Dermatol 2006; 15: 223

${ }^{8}$ Zouboulis CC, Turnbull JR, Martus P. Univariate and multivariate analyses comparing demographic, genetic, clinical, and serological risk factors for severe Adamantiades-Behçet's Disease. In: Zouboulis CC (ed). Adamantiades-Behçet's Disease. London, Amsterdam: Kluwer/ Plenum, 2003: 123-126

${ }^{9}$ Zouboulis CC, May T. Pathogenesis of Adamantiades-Behçet's disease. Med Microbiol Immunol 2003; 192: 149-155

${ }^{10}$ Zouboulis CC, Turnbull JR, Mühlradt PF. High seroprevalence of antiMycoplasma fermentans antibodies in patients with malignant aphthosis. J Invest Dermatol 2003; 121: $211-212$

${ }^{11}$ Altenburg A, Orawa H, Zouboulis CC. IL-18 and CRP but not IL-8 are sensitive course-indicators for acute clinical manifestations in Adamantiades-Behçet's disease. Exp Dermatol 2006; 15: 221

12 Zouboulis CC. Adamantiades-Behçet's disease. In: Katsambas AD, Lotti TM (eds). European Handbook of Dermatological Treatments, 2nd edition. Berlin, Heidelberg: Springer, 2003: 16-26 